\title{
Pneumocystis jirovecii
}

National Cancer Institute

\section{Source}

National Cancer Institute. Pneumocystis jirovecii. NCI Thesaurus. Code C117361.

A species of yeast-like fungus that is the causative organism of Pneumocystis

pneumonia. 